\title{
Suicide in older people - can it be prevented?
}

\author{
RC Baldwin \\ Manchester Royal Infirmary, Manchester, UK
}

\section{Editorial}

In the UK, one of the strategic aims for the health services is to reduce death by suicide. Older people are over-represented in national suicide figures in most developed countries so they are de facto a high risk group. For example, in the UK, people aged over 65 comprise about $15 \%$ of the population but this age group accounts for between 20 and $25 \%$ of all completed suicides.

Studies of suicide in older people consistently point to an association with depressive disorder in at least two-thirds of those who have killed themselves. ${ }^{1-3}$ There are less consistent data on possible links with other psychiatric disorders such as alcohol abuse and dementia. Personality factors have received less attention but disorders of personality are probably relatively uncommon among elderly suicide victims compared to younger ones.

Only a quarter or so of older people who kill themselves are known to psychiatric services although most will have been in recent contact with primary care services. ${ }^{3}$ So a strategy to reduce suicide which is aimed solely at patients with severe depression under psychiatric care will fail. Rather, identifying high-risk patients within primary care would appear to offer the best chance of reducing death by suicide in older people. But which factors predict who is at risk of suicide? Depressive disorder has already been mentioned and those depressed persons most at risk have a clinical picture characterized by severe agitation, guilt, hopelessness, insomnia and marked somatic preoccupation. Other factors which are associated with suicide in older people in the community are chronic painful conditions, functional limitation and social isolation. ${ }^{4}$ However, many more people live alone than ever attempt suicide. So rather than

Address for correspondence: Professor Robert C Baldwin, Manchester Mental Health Partnership, York House, Manchester Royal Infirmary, Oxford Rd, Manchester M13 9BX, UK. poor social circumstances contributing to suicide, a better model might be that better social conditions reduce vulnerability to it.

Caine $\mathrm{C}^{5}$ has outlined three broad approaches to suicide prevention in older people: health maintenance and promotion; treatment of depression in primary care and community settings; and screening for suicidal ideation, coupled with direct preventative efforts. With regard to the latter two strategies, the evidence of effectiveness is inconsistent. The Gotland study ${ }^{6}$ is the only one to date which has demonstrated that an educational programme to improve primary care physicians' knowledge of depression and its treatment can impact on suicide rates (in this case reducing the rate in women only). Intriguing though this is, it is unclear whether the findings can be generalized to other settings. In the USA, a major initiative is under way ('PROSPECT') which uses a 'guided management' intervention for depression, hopelessness and suicidal ideation within a collaborative care model. Data are awaited.

Clearly, appropriate detection and adequate management of depressive disorder, especially within primary care, remain a priority for suicide prevention in older people. However, Caine has argued for a rethink regarding suicide prevention, suggesting that a broad-based public health approach may be appropriate. Thus poor health, chronic pain, disablement and social isolation are the context for depression and suicide, just as poor diet, lack of exercise and smoking are for heart disease. Clearly, an approach to the prevention of mortality in heart disease which focussed exclusively on those with known ischaemic heart disease would not be acceptable. Perhaps it is time to rethink a 'healthy living' strategy for suicide prevention too. 


\section{References}

1 Barraclough BM. Suicide in the elderly. $\mathrm{Br} J$ Psychiatry 1971; 6: 87-97.

2 Cattell H, Jolley DJ. One hundred cases of suicide in elderly people. Br J Psychiatry 1995; 166: 451-57.

3 Harwood D, Hawrton, K. Hope T, Jacoby R. Psychiatric disorder and personality factors associated with suicide in older people: a descriptive and case-control study. Int J Geriatr Psychiatry 2001; 16: 155-65.

4 Conwell Y, Lyness, JM, Duberstein P et al.
Completed suicide among older patients in primary care practices: a controlled study. J Am Geriatr Soc 2000; 4823-29.

5 Caine ED. Presentation of the Joint Meeting of the International Psychogeriatric Association and the Faculty of Psychiatry of Old Age and Australian and New Zealand College of Psychiatrists; Lorne, Victoria Australia (Abstract p.30); February, 2001

6 Rutz W, yon Knorring L, Walinder J: Frequency of suicide on Gotland after systematic postgraduate education of general practitioners. Acta Psych Scand 1989; 80: 151-54. 